\title{
Projections for dark photon searches at Mu3e
}

\author{
Bertrand Echenard, ${ }^{a}$ Rouven Essig ${ }^{b}$ and Yi-Ming Zhong ${ }^{b}$ \\ ${ }^{a}$ California Institute of Technology, \\ Pasadena, California 91125, U.S.A. \\ ${ }^{b}$ C.N. Yang Institute for Theoretical Physics, Stony Brook University, \\ Stony Brook, NY 11794, U.S.A. \\ E-mail: echenard@hep.caltech.edu, rouven.essig@stonybrook.edu, \\ yiming.zhong@stonybrook.edu
}

ABstract: We show that dark photons $\left(A^{\prime}\right)$ with masses $\sim 10-80 \mathrm{MeV}$ can be probed in the decay $\mu^{+} \rightarrow e^{+} \nu_{e} \bar{\nu}_{\mu} A^{\prime}, A^{\prime} \rightarrow e^{+} e^{-}$, with the upcoming Mu3e experiment at the Paul Scherrer Institute (PSI) in Switzerland. With an expected $10^{15}\left(5.5 \times 10^{16}\right)$ muon decays in 2015-2016 (2018 and beyond), Mu3e has the exciting opportunity to probe a substantial fraction of currently unexplored dark photon parameter space, probing kineticmixing parameter, $\epsilon$, as low as $\epsilon^{2} \sim 10^{-7}\left(10^{-8}\right)$. No modifications of the existing Mu3e setup are required.

Keywords: Beyond Standard Model, Rare Decays, Electromagnetic Processes and Properties

ARXIV EPRINT: 1411.1770 


\section{Contents}

1 Introduction 1

2 The search for dark photons with Mu3e 3

3 Projections for dark photon searches with Mu3e 4

3.1 Promptly decaying dark photons 5

$\begin{array}{lll}3.2 & \text { Displaced vertices from dark photons } & 9\end{array}$

$\begin{array}{lll}4 & \text { Conclusions } & 10\end{array}$

$\begin{array}{ll}\text { A Estimation of accidental backgrounds } & 10\end{array}$

\section{Introduction}

There are only a few ways in which new particles and forces below the weak-scale can interact with the standard model (SM) particles and have remained undetected thus far. Among the simplest possibilities is the existence of a light, massive vector boson called a dark photon $\left(A^{\prime}\right)$. A substantial effort is underway to search for a dark photon with a variety of experiments. In this paper, we show that the upcoming Mu3e experiment at the Paul Scherrer Institute (PSI) in Switzerland is also sensitive to dark photons. Using an unprecedented number of muon decays ${ }^{1}$ in their search for the lepton flavor violating decay $\mu^{+} \rightarrow e^{+} e^{-} e^{+}, \mathrm{Mu} 3 \mathrm{e}$ can also search for the decay $\mu^{+} \rightarrow e^{+} \nu_{e} \bar{\nu}_{\mu} A^{\prime}, A^{\prime} \rightarrow e^{+} e^{-}$shown in figure 1. This allows them to probe currently unexplored regions of the dark photon parameter space. We note that while our focus will be on vector bosons (the dark photon), other particles that couple to electrons and/or muons and decay to an $e^{+} e^{-}$pair could also be probed with Mu3e.

The dark photon is the mediator of a new, broken $\mathrm{U}(1)_{\mathrm{D}}$ gauge group and appears in many theoretical scenarios, see e.g. [1-3] and references therein. It can interact with ordinary matter through "kinetic mixing" [4-6] with the SM hypercharge, $\mathrm{U}(1)_{\mathrm{Y}}$, gauge boson. At low energies, the dominant effect is a mixing of the $\mathrm{U}(1)_{\mathrm{D}}$ with the SM photon, $\mathrm{U}(1)_{\mathrm{EM}}$, as described with the Lagrangian

$$
\mathcal{L}=\mathcal{L}_{\mathrm{SM}}-\frac{\epsilon}{2} F_{\mu \nu}^{\prime} F^{\mu \nu}-\frac{1}{4} F_{\mu \nu}^{\prime} F^{\prime \mu \nu}+\frac{1}{2} m_{A^{\prime}}^{2} A_{\mu}^{\prime} A^{\prime \mu} .
$$

Here $\mathcal{L}_{\mathrm{SM}}$ is the SM Lagrangian, $\epsilon$ is the kinetic mixing parameter, $F^{\prime \mu \nu}\left(F^{\mu \nu}\right)$ is the $\mathrm{U}(1)_{\mathrm{D}}$ (U(1) $)_{\mathrm{EM}}$ ) field strength, and $m_{A^{\prime}}$ is the dark photon mass (the mechanism for generating this mass is not important for our purposes). The mixing between the dark photon and

\footnotetext{
1 "Muon" refers to $\mu^{+}$in this paper.
} 

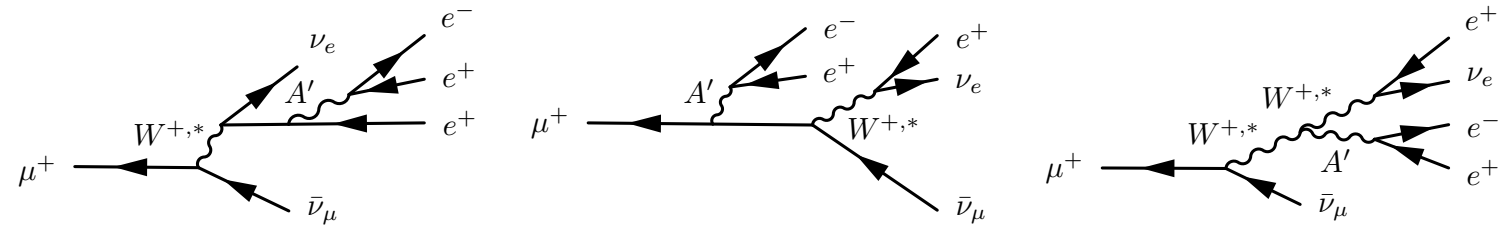

Figure 1. Feynman diagrams for (on-shell) dark photon production in muon decays, $\mu^{+} \rightarrow$ $e^{+} \nu_{e} \bar{\nu}_{\mu} A^{\prime}, A^{\prime} \rightarrow e^{-} e^{+}$.

the SM photon leads to an $\epsilon$-suppressed coupling of the dark photon to the electromagnetic current, $J_{\mathrm{EM}}^{\mu}$, i.e., to quarks and charged leptons,

$$
\mathcal{L} \supset \epsilon e A_{\mu}^{\prime} J_{\mathrm{EM}}^{\mu} .
$$

The two relevant parameters of the model are the kinetic mixing parameter and the dark photon mass. The coupling in eq. (1.2) allows the dark photon to be probed with a wide range of experiments, see e.g. [1-3] for a recent review and references. We do not consider the addition of other low-mass particles to this model.

Theoretically, the values of the kinetic mixing and the dark photon mass can take on a wide range of values. However, much attention has recently been focused on the $\mathrm{MeV}-\mathrm{GeV}$ mass range. In this mass range, the dark photon could explain the $\sim 3.6 \sigma$ discrepancy between the observed and SM value of the muon anomalous magnetic moment ( $a_{\mu} \equiv g_{\mu}-2$, where $g_{\mu}$ is the muon's gyromagnetic ratio) [7-9] and offer an explanation for various dark matter related anomalies through dark matter-dark photon interactions [1013]. Moreover, a dark photon mass in this range can be generated naturally in several new physics scenarios [14-18]. In addition, if $\mathrm{U}(1)_{\mathrm{Y}}$ is embedded in a Grand Unified Theory (GUT), the mixing can be generated by a one-(two-)loop interaction and naturally give $\epsilon \sim 10^{-3}-10^{-1}\left(\sim 10^{-5}-10^{-3}\right)[4,14,16,19]$.

There are many experimental probes of $\mathrm{MeV}-\mathrm{GeV}$ mass dark photons that decay directly to SM particles. These include collider experiments, beam dumps, rare meson decays, supernova cooling, and precision measurements [7, 18, 20-37, 37-50]. Existing constraints have almost disfavored the entire mass and coupling range in which dark photons could explain the mismatch between the observed and SM expected value of $a_{\mu}$, assuming the dark photon decays directly to SM particles with a branching ratio close to $100 \%$. A reduced branching ratio is possible if there exist other light particles that couple to the dark photon and open up additional decay modes.

In this paper, we will show that Mu3e can probe dark photons in the mass range $2 m_{e}<m_{A^{\prime}}<m_{\mu}$, where $m_{e}\left(m_{\mu}\right)$ is the electron (muon) mass, and improve upon current constraints on $\epsilon$ in the range $10 \mathrm{MeV} \lesssim m_{A^{\prime}} \lesssim 80 \mathrm{MeV}$, down to $\epsilon^{2} \sim 10^{-8}$. This probes well into the mentioned above parameter region motivated from embedding the $\mathrm{U}(1)_{\mathrm{Y}}$ in a GUT, as well as probing $a_{\mu}$ favored dark photon to SM branching ratios significantly less than 100\%. Depending on the performance of the detector, Mu3e may also be sensitive to long-lived dark photons, which produce displaced vertices.

The paper is organized as follows. We first summarize in section 2 how dark photons can be produced in muon decays and detected in Mu3e. We then discuss the projections 


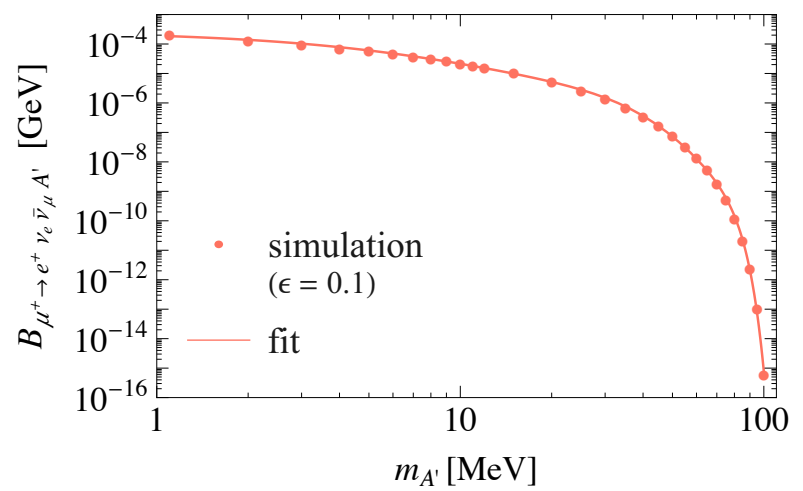

Figure 2. The branching ratio of the muon decay channel $\mu^{+} \rightarrow e^{+} \nu_{e} \bar{\nu}_{\mu} A^{\prime}$ with $\epsilon=0.1$. Shown are the numerical values computed with MadGraph5_aMC@NLO (red points) and a parametrized fit to these numerical values (red solid line), which is given by eq. (2.1).

for the sensitivity of dark photon searches at Mu3e for prompt decays in section 3.1 and briefly discuss a possible search using displaced vertices in section 3.2. Our conclusions are presented in section 4 . In appendix A, we detail our estimate of the accidental backgrounds for the prompt search.

\section{The search for dark photons with Mu3e}

The Mu3e experiment at PSI [51] has been proposed to search for the charged lepton flavor violating decay $\mu^{+} \rightarrow e^{+} e^{-} e^{+}$with an ultimate sensitivity of $10^{-16}$, four orders below the current limits. It will take advantage of one of the most intense sources of muons in the world. During its first phase (2015-2016), Mu3e will probe $10^{15}$ muon decays, and more than $5.5 \times 10^{16}$ muon decays by the end of phase II (2018 and beyond). To achieve the required sensitivity, a novel design based on high-granularity thin silicon pixel detectors, supplemented by a fast timing system, has been proposed.

The large statistics and excellent detector resolution offer an ideal setup to search for dark photon production in muon decays as well. The production mechanism is illustrated in figure 1: the dark photon can be either emitted from the initial state radiation off the $\mu^{+}$, or final state radiation off the $e^{+}$, or radiate off the internal $W$-boson. The latter process is suppressed by $\sim m_{\mu}^{2} / m_{W}^{2} \sim 10^{-6}$ at the amplitude level compared to the other processes due to the different propagators appearing in the diagrams (this is similar for the corresponding SM process where the dark photon is replaced by the SM photon, see also [52]). The corresponding decay width of $\mu^{+} \rightarrow e^{+} \nu_{e} \bar{\nu}_{\mu} A^{\prime}$, is evaluated using MadGraph5_aMC@NLO 2.1.0 [53] for $m_{A^{\prime}}$ ranging from $1.1 \mathrm{MeV}$ to $100 \mathrm{MeV}$. Approximating the total decay width, $\Gamma_{\text {tot }}$, as the SM muon decay width, the resulting branching ratio $B_{\text {sig }}$ is presented in figure 2 for $\epsilon=0.1$. We also include a parametrized curve (red, labeled "fit") of the form $B_{\text {sig }}=B_{\text {sig }}\left(\epsilon, m_{A^{\prime}}\right)$ with

$$
B_{\mathrm{sig}}=\frac{1}{3 \times 10^{-19}}\left(\frac{\epsilon}{0.1}\right)^{2} \exp \left(\sum_{i=0}^{5} a_{i}\left(\frac{m_{A^{\prime}}}{\mathrm{GeV}}\right)^{i}\right),
$$


where $a_{0}=-50.866, a_{1}=-360.93, a_{2}=13998.59, a_{3}=-3.731 \times 10^{5}, a_{4}=4.442 \times 10^{6}$, $a_{5}=-2.015 \times 10^{7}$, and we take the fine structure constant $\alpha=1 / 137.036$ and $\Gamma_{\text {tot }} \simeq$ $3 \times 10^{-19} \mathrm{GeV}[54]$.

For $2 m_{e} \leq m_{A^{\prime}} \leq 2 m_{\mu}$, the dominant decay is $A^{\prime} \rightarrow e^{+} e^{-}$(the loop-induced decay $A^{\prime} \rightarrow 3 \gamma$ is highly suppressed and only important for $m_{A^{\prime}}<2 m_{e}$ ). The main signature of such a dark photon is that the invariant mass of the $e^{+} e^{-}$-pair from the dark photon decay must equal $m_{A^{\prime}}$. The invariant mass spectrum is dominated by SM background events (see section 3), but a resonance search or "bump-hunt" can be used to search for a dark photon.

In addition to a resonance search, displaced $e^{+} e^{-}$vertices can also be used to probe long-lived dark photons. The dark photon width and the proper decay length are given by, respectively,

$$
\begin{aligned}
\Gamma_{A^{\prime} \rightarrow e^{+} e^{-}} & =\frac{\alpha \epsilon^{2}}{3} m_{A^{\prime}} \sqrt{1-\frac{4 m_{e}^{2}}{m_{A^{\prime}}^{2}}}\left(1+\frac{2 m_{e}^{2}}{m_{A^{\prime}}^{2}}\right), \\
c \tau_{A^{\prime} \rightarrow e^{+} e^{-}} & \simeq 0.8 \mathrm{~mm}\left(\frac{10^{-4}}{\epsilon}\right)^{2} \frac{10 \mathrm{MeV}}{m_{A^{\prime}}} .
\end{aligned}
$$

For small-enough values of $\epsilon$, the dark photon will travel a finite distance and the $e^{+} e^{-}$-pair will be reconstructed as a displaced vertex (for even smaller values, the decay length will be large enough to allow for the shielding of almost any backgrounds, as in beam-dump experiments). Since the backgrounds are expected to be greatly reduced with respect to prompt decays, displaced vertices could provide sensitivity to low values of the kinematic mixing.

\section{Projections for dark photon searches with Mu3e}

The sensitivity to dark photons with an experimental setup similar to that of Mu3e is studied using a simulation program, FastSim, that was originally developed for the Super $B$ experiment [55], based on the software framework and analysis tools used by the BABAR collaboration [56, 57]. Detector components are described in FastSim as two-dimensional shells of geometric objects, such as cylinders, disks, or planes, and the effect of the physical thickness is modeled parametrically. Coulomb scattering and energy loss by ionization are described with the standard parametrization in terms of radiation length and particle momentum. Simplified cross sections are used to describe Bremsstrahlung and pair production. Tracking measurements are simulated in terms of single-hit and two-hit resolutions, while silicon strip detectors are modeled as two independent orthogonal projections. Tracks are reconstructed from the simulated hits passed to the BABAR Kalman filter track fitting algorithm. Uncertainties associated with pattern recognition algorithms traditionally used to form track hits are introduced using models based on the BABAR pattern recognition algorithm performance.

The FastSim model is a simplified version of the proposed Mu3e detector [51], which consists of a silicon tracker composed of two inner and two outer double layers of cylindrical pixel silicon detectors surrounding the target. The inner layers have a length of $12 \mathrm{~cm}$, 

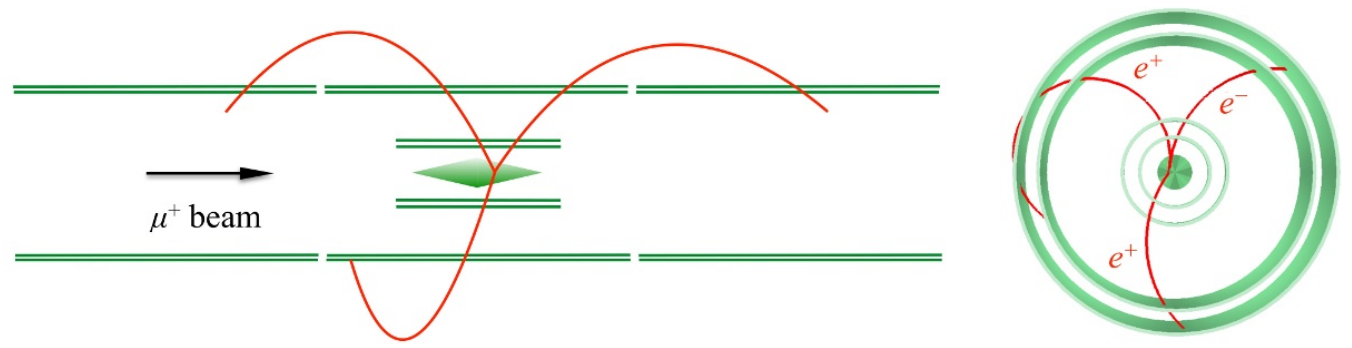

Figure 3. Left: side view of the experimental setup (adapted from [51]). A muon beam impinges on a target consisting of two hollow aluminum cones connected at their base. A silicon tracker composed of two inner and two outer double layers of cylindrical pixel silicon surrounds the target. Although not included in the simulation, a time-of-flight device provides a timing measurement with a resolution of 250 ps. A simulated $\mu^{+} \rightarrow e^{+} \nu_{e} \bar{\nu}_{\mu} A^{\prime}, A^{\prime} \rightarrow e^{+} e^{-}$event is shown. Right: transverse view of the experimental setup. The stopping target is shown at the center, surrounded by two inner and two outer cylindrical layers of silicon detectors.

while the outer silicon layers are extended to a length of $180 \mathrm{~cm}$ to improve the momentum resolution of recurling tracks. The innermost (outermost) silicon detectors are placed at a radius of $1.9 \mathrm{~cm}(8.9 \mathrm{~cm})$. Silicon sensors are simulated as $50 \mu \mathrm{m}$ thick double-sided striplet sensors mounted on $50 \mu \mathrm{m}$ of kapton in FastSim. The spatial resolution of the hits is modeled as a sum of two components with resolutions of $8 \mu \mathrm{m}$ and $20 \mu \mathrm{m}$. Although Mu3e uses pixel silicon sensors, we expect the performances of both tracking system to be comparable. The target is composed of two hollow aluminum cones connected at their base. Each cone is $5 \mathrm{~cm}$ long, $50 \mu \mathrm{m}$ thick with a base radius of $1 \mathrm{~cm}$. The entire detector is placed in a $1 \mathrm{~T}$ solenoidal magnetic field. Although not included in FastSim, a timeof-flight device provides a timing measurement. We assume a time resolution of $250 \mathrm{ps,}$ averaging the values of the corresponding Mu3e detector systems. We define a coordinate system having the $z$-axis aligned along the axis of the cylindrical silicon detectors, with the transverse plane oriented perpendicular to the $z$-axis. The apparatus layout is displayed in figure 3 , together with a simulated $\mu^{+} \rightarrow e^{+} \nu_{e} \bar{\nu}_{\mu} A^{\prime}, A^{\prime} \rightarrow e^{+} e^{-}$event.

\subsection{Promptly decaying dark photons}

We begin by studying the sensitivity of prompt dark photon decays in $\mu^{+} \rightarrow e^{+} \nu_{e} \bar{\nu}_{\mu} A^{\prime}$, $A^{\prime} \rightarrow e^{+} e^{-}$events. Large samples of signal and background events are generated to study the signal efficiency and background levels. We assume that muons decay uniformly at rest in the target. Signal events are generated with MadGraph5_aMC@NLO 2.1.0 for $5 \mathrm{MeV}<m_{A^{\prime}}<100 \mathrm{MeV}$. The background processes can be classified as either irreducible or accidental:

- Irreducible backgrounds arise from events with internal conversions of the photon in $\mu^{+} \rightarrow e^{+} \nu_{e} \bar{\nu}_{\mu} \gamma^{*}\left(\rightarrow e^{+} e^{-}\right)$decays, or from radiative muon decays where the radiated photon converts into an electron-positron pair inside the target material. Conversion outside the target material, i.e., in the detector material, can be efficiently tagged and are not considered. These background processes are simulated using the matrix 
element and differential decay width given in $[52,58]$, and the events are normalized using the following branching fractions: $B_{\mu^{+} \rightarrow e^{+} \nu_{e} \bar{\nu}_{\mu} e^{+} e^{-}}=(3.4 \pm 0.4) \times 10^{-5}$ and $B_{\mu^{+} \rightarrow e^{+} \nu_{e} \bar{\nu}_{\mu} \gamma}=(1.4 \pm 0.4) \%$ [54]. As the probability of photon conversion inside the target is of $\mathcal{O}\left(10^{-3}\right)$, both channels contribute roughly equally to the irreducible background.

- Accidental backgrounds arise mainly from the combination of several muon decays where, e.g., one of the positrons is misreconstructed as an electron. We consider background sources from the following accidental combinations: (1) three Michel decays $\left(\mu^{+} \rightarrow e^{+} \nu_{e} \bar{\nu}_{\mu}\right)$ where one positron is misreconstructed as an electron ("3M decays"), (2) a Michel decay and a radiative Michel decay $\left(\mu^{+} \rightarrow e^{+} \nu_{e} \bar{\nu}_{\mu} \gamma\right)$ where the photon converts to a $e^{+} e^{-}$pair in the target material and one electron remains undetected (" $2 \mathrm{M}_{\gamma}$ decays"), and (3) a Michel decay and a radiative Michel decay with internal conversion where one electron again remains undetected (" $2 \mathrm{M}_{3 e}$ decays"). Another source of accidental background, which we will not include, arises from two Michel decays where the outgoing positron from one of the Michel decays produces an electron by interacting with the detector material via Bhabha scattering (" $2 \mathrm{M}_{\text {Bhabha }}$ decays"). Other sources of accidental backgrounds are expected to be smaller.

We generate signal and background events and process them with FastSim to determine the detection efficiency and the invariant mass distribution, $m_{e^{+} e^{-}}$. We require all electrons and positrons to have a minimum transverse momentum of $10 \mathrm{MeV}$ to match the $\mathrm{Mu} 3 \mathrm{e}$ tracker acceptance. The $\mu^{+} \rightarrow e^{+} \nu_{e} \bar{\nu}_{\mu} e^{+} e^{-}$candidates are formed by combining two positrons and an electron, and fit with the constraint that the tracks originate from the same position at the surface of the target. We select only well reconstructed candidates by requiring the probability of the $\chi^{2}$ of the constrained fit to be greater than $1 \%$. Additional kinematic constraints can further distinguish $\mu^{+} \rightarrow e^{+} \nu_{e} \bar{\nu}_{\mu} e^{+} e^{-}$decays from accidental backgrounds. The magnitude of the sum of the momenta of the electron and two positrons $\left(\left|\vec{p}_{3 e}\right| \equiv\left|\vec{p}_{e^{-}}+\vec{p}_{e^{+}, 1}+\vec{p}_{e^{+}, 2}\right|\right)$ must be compatible with the muon decay hypothesis, requiring

$$
\left|\vec{p}_{3 e}\right| \leq \frac{m_{\mu}^{2}-m_{3 e}^{2}}{2 m_{\mu}},
$$

where $m_{3 e}$ is the invariant mass of the three tracks.

While we can reliably determine the efficiency of the signal and irreducible backgrounds, it is more challenging to estimate the accidental backgrounds with very high accuracy. However, the following approach, which is similar to that described by Mu3e [51], is sufficient for our purposes. The number of $3 \mathrm{M}, 2 \mathrm{M}_{\gamma}$, and $2 \mathrm{M}_{3 e}$ decays are given by, respectively,

$$
\begin{aligned}
N_{3 \mathrm{M}} & \simeq \frac{1}{2} N_{\mu} R_{\mu}^{2} \delta t^{2} P_{\mathrm{p}}^{2} B_{\mu^{+} \rightarrow e^{+} \nu_{e} \bar{\nu}_{\mu}}^{3} P_{e^{+} \rightarrow e^{-}}, \\
N_{2 \mathrm{M}_{\gamma}} & \simeq N_{\mu} R_{\mu} \delta t P_{\mathrm{p}} B_{\mu^{+} \rightarrow e^{+} \nu_{e} \bar{\nu}_{\mu}} B_{\mu^{+} \rightarrow e^{+} \nu_{e} \bar{\nu}_{\mu} \gamma} P_{\gamma}, \\
N_{2 \mathrm{M}_{3 e}} & \simeq N_{\mu} R_{\mu} \delta t P_{\mathrm{p}} B_{\mu^{+} \rightarrow e^{+} \nu_{e} \bar{\nu}_{\mu}} B_{\mu^{+} \rightarrow e^{+} \nu_{e} \bar{\nu}_{\mu} e^{+} e^{-}}
\end{aligned}
$$



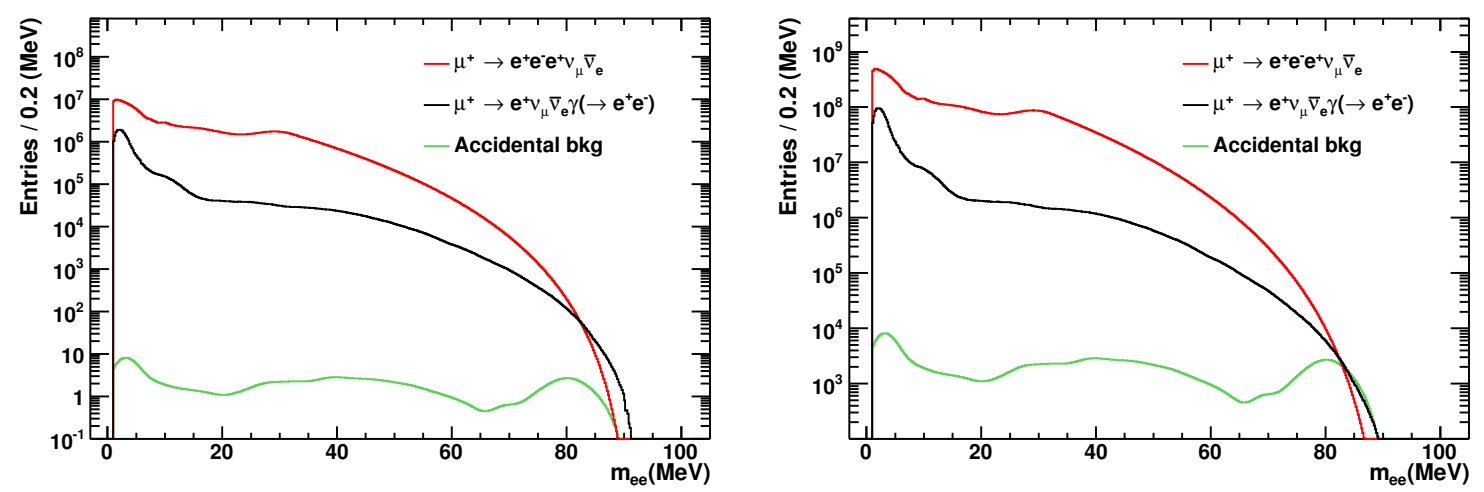

Figure 4. The expected $e^{+} e^{-}$invariant mass distribution from the various sources of background displayed assuming a total number of $10^{15}$ (left) or $5.5 \times 10^{16}$ (right) muon decays for the phase I and II of Mu3e, respectively. The accidental backgrounds include the $3 \mathrm{M}, 2 \mathrm{M}_{\gamma}$, and $2 \mathrm{M}_{3 e}$ backgrounds but not the $2 \mathrm{M}_{\text {Bhabha }}$ background (see text for details).

where $N_{\mu}=10^{15}\left(2 \times 10^{16}\right)$ is the total number of muons and $R_{\mu}=10^{8} / \mathrm{s}\left(2 \times 10^{9} / \mathrm{s}\right)$ is the instantaneous stopped muon rate for Mu3e phase I (II), $\delta t=2.5 \times 10^{-10} \mathrm{~s}$ is the average time resolution, $P_{\mathrm{p}}=10^{-4}$ is the position suppression factor, $P_{e^{+} \rightarrow e^{-}}=0.5 \%$ is the positron-to-electron misidentification probability, and $P_{\gamma}=8 \times 10^{-4}$ is the photon conversion probability in the target. Derivations of eqs. (3.2), (3.3) and (3.4) are shown in appendix A. Inserting the numbers, we find that the expected number of accidental background events over the lifetime of the experiment (before correcting for the efficiency) are given by, roughly, $N_{3 \mathrm{M}} \sim 15,000(60,000), N_{2 \mathrm{M}_{\gamma}} \sim 30,000\left(6 \times 10^{6}\right)$, and $N_{2 \mathrm{M}_{\mathrm{e}}} \sim$ $75,000\left(2 \times 10^{7}\right)$ for phase I (II). We use these numbers to normalize each accidental background component. We note that we will not consider the $2 \mathrm{M}_{\text {Bhabha }}$ background, as it is challenging to simulate reliably. More study is needed by the Mu3e Collaboration to determine its size, but preliminary estimates ${ }^{2}$ suggest that in the $10 \mathrm{MeV}$ to $80 \mathrm{MeV}$ mass range, this background should be at most comparable, but more likely subdominant, to the irreducible backgrounds.

The $e^{+} e^{-}$invariant mass distribution of the most important irreducible and accidental backgrounds, after applying all selection criteria, is shown in figure 4, assuming a total number of $10^{15}$ (left plot) and $5.5 \times 10^{16}$ (right plot) muon decays for the two phases of Mu3e, respectively. Both combinations per muon candidate are considered and included in the corresponding histograms. The signal reconstruction efficiency is shown in figure 5 and varies between $7 \%$ and $41 \%$, depending on the dark photon mass.

As expected, the distribution peaks towards low values of $m_{e^{+} e^{-}}$. The spectrum is dominated by $\mu^{+} \rightarrow e^{+} \nu_{e} \bar{\nu}_{\mu} e^{+} e^{-}$events (red line in figure 4) with an additional contribution from $\mu^{+} \rightarrow e^{+} \nu_{e} \bar{\nu}_{\mu} \gamma$ with the conversion $\gamma \rightarrow e^{+} e^{-}$in the target material (black line). The accidental backgrounds (green line) are subdominant, except for $m_{e^{+} e^{-}} \gtrsim 80 \mathrm{MeV}$, where they become comparable to the irreducible contribution. However, as we will discuss below, this region is already well explored by existing experiments. Therefore, even

\footnotetext{
${ }^{2}$ We thank André Schöning for valuable discussions of this background.
} 


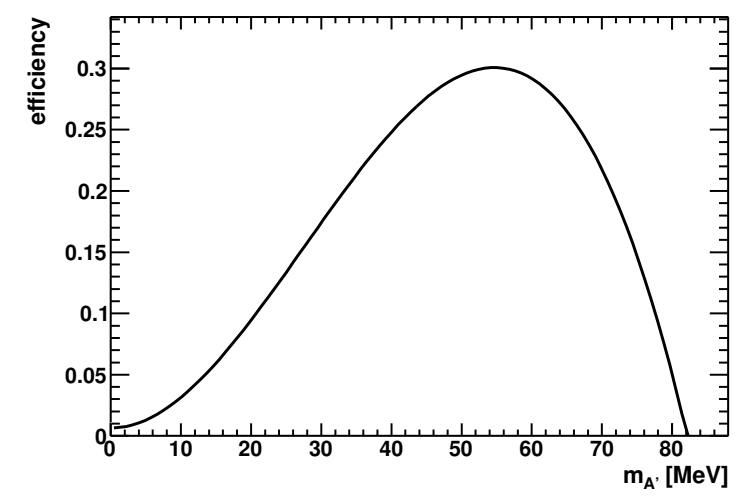

Figure 5. The signal efficiency as a function of the dark photon mass $\left(m_{A^{\prime}}\right)$ for prompt $\mu^{+} \rightarrow$ $e^{+} \nu_{e} \bar{\nu}_{\mu} A^{\prime}, A^{\prime} \rightarrow e^{+} e^{-}$decays.

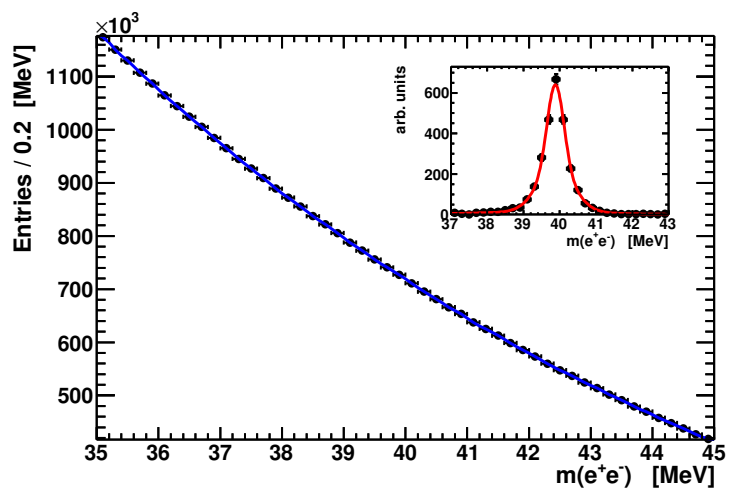

Figure 6. Fit to the $e^{+} e^{-}$invariant mass distribution for a dark photon mass hypothesis of $40 \mathrm{MeV}$. The blue line shows the expected background. The signal probability density function, as obtained from a fit to the signal Monte Carlo sample, is shown as the red line in the insert.

if our accidental background estimate is off by a factor of a few, it will have little impact on the the dark-photon parameter region probed by Mu3e that is currently unexplored $\left(m_{A^{\prime}} \lesssim 70 \mathrm{MeV}\right)$.

A dark photon signal would appear as a narrow peak over the smooth background distribution. The signal resolution is determined by fitting the corresponding mass spectrum with a sum of three Gaussians. The central mass resolution is at the level of $0.2-0.3 \mathrm{MeV}$, almost independent of $m_{A^{\prime}}$. We checked that these results are similar to the expected performance of the Mu3e detector [51].

We estimate the signal sensitivity by fitting a signal component on top of the expected background in the range $10 \mathrm{MeV}<m_{A^{\prime}}<80 \mathrm{MeV}$. Each fit is performed over an interval of $\pm 5 \mathrm{MeV}$ around the nominal dark photon mass. An example of a fit is displayed in figure 6 . We extract a $95 \%$ confidence level (CL) limit on the number of signal events, and derive a bound on the $\mu^{+} \rightarrow e^{+} \nu_{e} \bar{\nu}_{\mu} A^{\prime}, A^{\prime} \rightarrow e^{+} e^{-}$branching fraction by dividing by the signal efficiency and the number of muon decays. These results are translated into limits on the kinetic mixing parameter, $\epsilon$, and shown as a blue (red) solid line for Mu3e's 


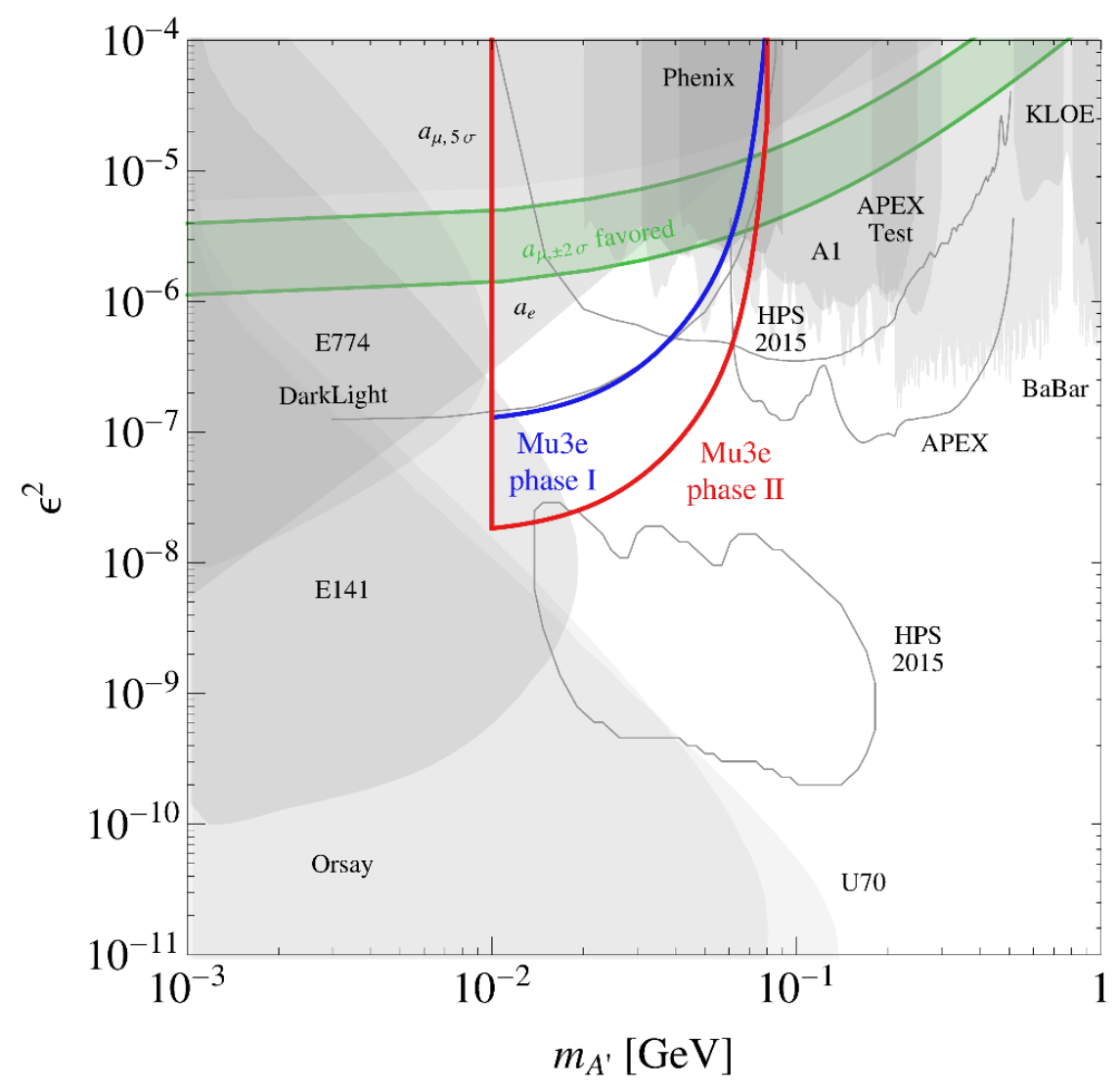

Figure 7. Prospects and constraints in the $\epsilon^{2}$ versus $m_{A^{\prime}}$ plane for dark photons that decay directly to SM particles (see e.g. [1] and references given in section 1 of this paper). The projected sensitivity of a resonance search for promptly decaying dark photons with the Mu3e experiment is shown in blue (red) assuming $10^{15}\left(5.5 \times 10^{16}\right)$ muon decays for Mue3 phase I (II).

phase I (II) in figure 7, together with existing constraints and prospects for upcoming experiments.

A substantial fraction of open parameter space in the low $m_{A^{\prime}}$ region can be explored, complementing or overlapping the reach of currently planned experiments, including APEX [28, 36], HPS [47], DarkLight [27, 43], and an experiment at the SPS [46] (the latter is not shown). As mentioned in the introduction, if $\mathrm{U}(1)_{\mathrm{Y}}$ is embedded in a GUT, the mixing that is generated by a one-(two-)loop interaction naturally gives $\epsilon^{2} \sim 10^{-6}-10^{-2}$ $\left(\sim 10^{-10_{-}}-10^{-6}\right)$. Mu3e has the opportunity to explore part of this theoretically interesting parameter space.

\subsection{Displaced vertices from dark photons}

For sufficiently small values of $\epsilon$, the dark photon lifetime can be sizable (see eq. (2.3)), leading to displaced decay vertices observable in the laboratory frame. While smaller values of $\epsilon$ lead to smaller muon branching fractions to dark photons, the backgrounds associated with displaced vertices are substantially reduced, providing an opportunity to observe a signal. The discovery potential depends on the geometrical acceptance of the detector, 
the vertex resolution, and the backgrounds. The assumptions introduced in the prompt decay scenario to treat the accidental backgrounds, i.e., pile-up events arising from muons decaying within the same time window and at the same position in the target, might not be valid anymore for displaced vertices. A full analysis should include contributions from pileup of several (radiative) muon decays generated everywhere in the target, which is beyond the scope of our paper, given the large number of muon decays involved. Furthermore, a small residual background from misreconstructed $\mu^{+} \rightarrow e^{+} \nu_{e} \bar{\nu}_{\mu} e^{+} e^{-}$events is expected to remain, and the accuracy of FastSim might be too limited to reliably predict its level. We encourage the Mu3e Collaboration to perform a detailed reach estimate, both because the tools at our disposal are not sufficient for a reliable estimate and because the sensitivity that could potentially be achieved is well worth the effort.

\section{Conclusions}

We have studied the possibility to search for dark photon in $\mu^{+} \rightarrow e^{+} \nu_{e} \bar{\nu}_{\mu} A^{\prime}, A^{\prime} \rightarrow e^{+} e^{-}$ decays with an apparatus similar to the Mu3e experiment. We derive sensitivity estimates for both prompt and displaced dark photon decays. Mu3e has the exciting opportunity to probe a substantial fraction of currently unexplored parameter space in the mass range $10 \mathrm{MeV} \lesssim m_{A^{\prime}} \lesssim 80 \mathrm{MeV}$ for $\epsilon^{2} \gtrsim 10^{-8}$, using a resonance search, overlapping or complementing the reach of currently planned experiments. This opportunity does not require any modifications of their existing setup. A search for displaced vertices may have sensitivity to lower values of $\epsilon$, but the precise reach estimate depends on the backgrounds, which require a careful modeling by the Mu3e Collaboration.

\section{Acknowledgments}

We thank André Schöning for reading and providing useful comments on the manuscript. BE is supported by the U.S. Department of Energy (DoE) under grant DE-FG02-92ER40701 and DE-SC0011925. RE and YZ are supported by the DoE Early Career research program DESC0008061. RE acknowledges additional support through a Sloan Foundation Research Fellowship.

\section{A Estimation of accidental backgrounds}

In section 3.1, we provided an estimate of the accidental backgrounds, see eqs. (3.2), (3.3) and (3.4). In this appendix, we justify these equations.

We first estimate the accidental background arising from three Michel decays, where one positron is misreconstructed as an electron $\left(N_{3 \mathrm{M}}\right)$. We assume a measurement takes place in a time interval $T \gg \delta t$, where $\delta t$ is the time resolution. The probability for three decays to occur in the same time window is $(\delta t / T)^{2}$ and at the same position is $P_{\mathrm{p}}^{2}$, where $P_{\mathrm{p}}$ is the position suppression factor. Multiplying these probabilities with the branching ratio for three Michel decays, $B_{\mu^{+} \rightarrow e^{+} \nu_{e} \bar{\nu}_{\mu}}^{3}$ and the probability for one out of three positrons 
to be misreconstructed as an electron, $P_{e^{+} \rightarrow e^{-}}$, the total probability for the $3 \mathrm{M}$ pile-up is given by

$$
P_{3 \mathrm{M}}=\left(\frac{\delta t}{T}\right)^{2} P_{\mathrm{p}}^{2} B_{\mu^{+} \rightarrow e^{+} \nu_{e} \bar{\nu}_{\mu}}^{3}\left(\begin{array}{l}
3 \\
1
\end{array}\right) P_{e^{+} \rightarrow e^{-}} .
$$

During the time $T$, the number of stopped muons, $N_{\mu} \equiv R_{\mu} T$, and the number of $3 \mathrm{M}$ pile-up event, $N_{3 \mathrm{M}}$, are related by

$$
N_{3 \mathrm{M}}=\left(\begin{array}{c}
N_{\mu} \\
3
\end{array}\right) P_{3 \mathrm{M}} .
$$

Substituting eq. (A.1) into eq. (A.2) yields

$$
\begin{aligned}
N_{3 \mathrm{M}} & =\frac{1}{2} T R_{\mu}\left(R_{\mu}-\frac{1}{T}\right)\left(R_{\mu}-\frac{2}{T}\right) \delta t^{2} P_{\mathrm{p}}^{2} B_{\mu^{+} \rightarrow e^{+} \nu_{e} \bar{\nu}_{\mu}}^{3} \\
& \simeq \frac{1}{2} N_{\mu} R_{\mu}^{2} \delta t^{2} P_{\mathrm{p}}^{2} B_{\mu^{+} \rightarrow e^{+} \nu_{e} \bar{\nu}_{\mu}}^{3}
\end{aligned}
$$

which is eq. (3.2). The approximation is valid in the limit $R_{\mu} T \gg 1$.

Similarly, the total probabilities for the $2 \mathrm{M}_{\gamma}$ and $2 \mathrm{M}_{3 e}$ accidental backgrounds are given by

$$
\begin{aligned}
P_{2 \mathrm{M}_{\gamma}} & =\left(\begin{array}{l}
2 \\
1
\end{array}\right) B_{\mu^{+} \rightarrow e^{+} \nu_{e} \bar{\nu}_{\mu}} B_{\mu^{+} \rightarrow e^{+} \nu_{e} \bar{\nu}_{\mu} \gamma} P_{\gamma}, \\
P_{2 \mathrm{M}_{3 e}} & =\left(\begin{array}{l}
2 \\
1
\end{array}\right) B_{\mu^{+} \rightarrow e^{+} \nu_{e} \bar{\nu}_{\mu}} B_{\mu^{+} \rightarrow e^{+} \nu_{e} \bar{\nu}_{\mu} e^{+} e^{-}}
\end{aligned}
$$

respectively. Given the number of $2 \mathrm{M}_{\gamma}$ and $2 \mathrm{M}_{3 e}$ as

$$
\begin{aligned}
N_{2 \mathrm{M}_{\gamma}} & =\left(\begin{array}{c}
N_{\mu} \\
2
\end{array}\right) P_{2 \mathrm{M}_{\gamma}}, \\
N_{2 \mathrm{M}_{3 e}} & =\left(\begin{array}{c}
N_{\mu} \\
2
\end{array}\right) P_{2 \mathrm{M}_{3 e}},
\end{aligned}
$$

one finds

$$
\begin{aligned}
N_{2 \mathrm{M}_{\gamma}} & =T R_{\mu}\left(R_{\mu}-\frac{1}{T}\right) \delta t P_{\mathrm{p}} B_{\mu^{+} \rightarrow e^{+} \nu_{e} \bar{\nu}_{\mu}} B_{\mu^{+} \rightarrow e^{+} \nu_{e} \bar{\nu}_{\mu} \gamma} P_{\gamma} \\
& \simeq T R_{\mu}^{2} \delta t P_{\mathrm{p}} B_{\mu^{+} \rightarrow e^{+} \nu_{e} \bar{\nu}_{\mu}} B_{\mu^{+} \rightarrow e^{+} \nu_{e} \bar{\nu}_{\mu} \gamma} P_{\gamma}, \\
N_{2 \mathrm{M}_{3 e}} & =T R_{\mu}\left(R_{\mu}-\frac{1}{T}\right) \delta t P_{\mathrm{p}} B_{\mu^{+} \rightarrow e^{+} \nu_{e} \bar{\nu}_{\mu}} B_{\mu^{+} \rightarrow e^{+} \nu_{e} \bar{\nu}_{\mu} e^{+} e^{-}} \\
& \simeq T R_{\mu}^{2} \delta t P_{\mathrm{p}} B_{\mu^{+} \rightarrow e^{+} \nu_{e} \bar{\nu}_{\mu}} B_{\mu^{+} \rightarrow e^{+} \nu_{e} \bar{\nu}_{\mu} e^{+} e^{-}} .
\end{aligned}
$$

These are eqs. (3.3) and (3.4). The approximations in the above equations are again valid for $R_{\mu} T \gg 1$. 
Open Access. This article is distributed under the terms of the Creative Commons Attribution License (CC-BY 4.0), which permits any use, distribution and reproduction in any medium, provided the original author(s) and source are credited.

\section{References}

[1] R. Essig et al., Dark sectors and new, light, weakly-coupled particles, arXiv:1311.0029 [INSPIRE].

[2] J.L. Hewett et al., Fundamental physics at the intensity frontier, arXiv:1205.2671 [INSPIRE].

[3] J. Jaeckel and A. Ringwald, The low-energy frontier of particle physics, Ann. Rev. Nucl. Part. Sci. 60 (2010) 405 [arXiv: 1002.0329] [InSPIRE].

[4] B. Holdom, Two U(1)'s and $\epsilon$ charge shifts, Phys. Lett. B 166 (1986) 196 [INSPIRE].

[5] P. Galison and A. Manohar, Two Z's or not two Z's?, Phys. Lett. B 136 (1984) 279 [INSPIRE].

[6] K.R. Dienes, C.F. Kolda and J. March-Russell, Kinetic mixing and the supersymmetric gauge hierarchy, Nucl. Phys. B 492 (1997) 104 [hep-ph/9610479] [INSPIRE].

[7] M. Pospelov, Secluded U(1) below the weak scale, Phys. Rev. D 80 (2009) 095002 [arXiv:0811.1030] [INSPIRE].

[8] Muon G-2 collaboration, G.W. Bennett et al., Final report of the E821 muon anomalous magnetic moment measurement at BNL, Phys. Rev. D 73 (2006) 072003 [hep-ex/0602035] [INSPIRE].

[9] M. Davier, A. Hoecker, B. Malaescu and Z. Zhang, Reevaluation of the hadronic contributions to the muon $g-2$ and to $\alpha\left(M_{Z}^{2}\right)$, Eur. Phys. J. C 71 (2011) 1515 [Erratum ibid. C 72 (2012) 1874] [arXiv:1010.4180] [INSPIRE].

[10] N. Arkani-Hamed, D.P. Finkbeiner, T.R. Slatyer and N. Weiner, A theory of dark matter, Phys. Rev. D 79 (2009) 015014 [arXiv: 0810.0713] [INSPIRE].

[11] M. Pospelov and A. Ritz, Astrophysical signatures of secluded dark matter, Phys. Lett. B 671 (2009) 391 [arXiv:0810.1502] [INSPIRE].

[12] D.P. Finkbeiner and N. Weiner, Exciting dark matter and the INTEGRAL/SPI $511 \mathrm{keV}$ signal, Phys. Rev. D 76 (2007) 083519 [astro-ph/0702587] [INSPIRE].

[13] P. Fayet, Light spin 1/2 or spin 0 dark matter particles, Phys. Rev. D 70 (2004) 023514 [hep-ph/0403226] [INSPIRE].

[14] N. Arkani-Hamed and N. Weiner, LHC signals for a superunified theory of dark matter, JHEP 12 (2008) 104 [arXiv:0810.0714] [INSPIRE].

[15] C. Cheung, J.T. Ruderman, L.-T. Wang and I. Yavin, Kinetic mixing as the origin of light dark scales, Phys. Rev. D 80 (2009) 035008 [arXiv:0902.3246] [InSPIRE].

[16] M. Baumgart, C. Cheung, J.T. Ruderman, L.-T. Wang and I. Yavin, Non-Abelian dark sectors and their collider signatures, JHEP 04 (2009) 014 [arXiv: 0901.0283] [INSPIRE].

[17] D.E. Morrissey, D. Poland and K.M. Zurek, Abelian hidden sectors at a GeV, JHEP 07 (2009) 050 [arXiv: 0904.2567] [INSPIRE].

[18] R. Essig, P. Schuster and N. Toro, Probing dark forces and light hidden sectors at low-energy $e^{+} e^{-}$colliders, Phys. Rev. D 80 (2009) 015003 [arXiv: 0903.3941] [INSPIRE]. 
[19] R. Essig, J. Kaplan, P. Schuster and N. Toro, On the origin of light dark matter species, arXiv: 1004.0691 [INSPIRE].

[20] J.D. Bjorken, R. Essig, P. Schuster and N. Toro, New fixed-target experiments to search for dark gauge forces, Phys. Rev. D 80 (2009) 075018 [arXiv:0906.0580] [INSPIRE].

[21] J.D. Bjorken et al., Search for neutral metastable penetrating particles produced in the SLAC beam dump, Phys. Rev. D 38 (1988) 3375 [InSPIRE].

[22] E.M. Riordan et al., A search for short lived axions in an electron beam dump experiment, Phys. Rev. Lett. 59 (1987) 755 [INSPIRE].

[23] A. Bross et al., A search for short-lived particles produced in an electron beam dump, Phys. Rev. Lett. 67 (1991) 2942 [INSPIRE].

[24] B. Batell, M. Pospelov and A. Ritz, Probing a secluded U(1) at B-factories, Phys. Rev. D 79 (2009) 115008 [arXiv: 0903. 0363] [INSPIRE].

[25] M.J. Strassler and K.M. Zurek, Echoes of a hidden valley at hadron colliders, Phys. Lett. B 651 (2007) 374 [hep-ph/0604261] [INSPIRE].

[26] M.J. Strassler, Possible effects of a hidden valley on supersymmetric phenomenology, hep-ph/0607160 [INSPIRE].

[27] M. Freytsis, G. Ovanesyan and J. Thaler, Dark force detection in low energy e-p collisions, JHEP 01 (2010) 111 [arXiv:0909.2862] [INSPIRE].

[28] R. Essig, P. Schuster, N. Toro and B. Wojtsekhowski, An electron fixed target experiment to search for a new vector boson $A^{\prime}$ decaying to $e^{+} e^{-}$, JHEP 02 (2011) 009 [arXiv:1001.2557] [INSPIRE].

[29] J. Blumlein and J. Brunner, New exclusion limits for dark gauge forces from beam-dump data, Phys. Lett. B 701 (2011) 155 [arXiv:1104.2747] [InSPIRE].

[30] S. Andreas, C. Niebuhr and A. Ringwald, New limits on hidden photons from past electron beam dumps, Phys. Rev. D 86 (2012) 095019 [arXiv:1209.6083] [InSPIRE].

[31] M. Reece and L.-T. Wang, Searching for the light dark gauge boson in GeV-scale experiments, JHEP 07 (2009) 051 [arXiv:0904.1743] [INSPIRE].

[32] BABAR collaboration, B. Aubert et al., Search for dimuon decays of a light scalar boson in radiative transitions $\Upsilon \rightarrow \gamma A^{0}$, Phys. Rev. Lett. 103 (2009) 081803 [arXiv:0905.4539] [inSPIRE].

[33] A. Hook, E. Izaguirre and J.G. Wacker, Model independent bounds on kinetic mixing, Adv. High Energy Phys. 2011 (2011) 859762 [arXiv: 1006.0973] [INSPIRE].

[34] KLOE-2 collaboration, D. Babusci et al., Limit on the production of a light vector gauge boson in $\phi$ meson decays with the KLOE detector, Phys. Lett. B 720 (2013) 111 [arXiv: 1210.3927] [INSPIRE].

[35] F. Archilli et al., Search for a vector gauge boson in phi meson decays with the KLOE detector, Phys. Lett. B 706 (2012) 251 [arXiv:1110.0411] [INSPIRE].

[36] APEX collaboration, S. Abrahamyan et al., Search for a new gauge boson in electron-nucleus fixed-target scattering by the APEX experiment, Phys. Rev. Lett. 107 (2011) 191804 [arXiv:1108.2750] [INSPIRE].

[37] H. Merkel et al., Search at the Mainz Microtron for light massive gauge bosons relevant for the muon g- 2 anomaly, Phys. Rev. Lett. 112 (2014) 221802 [arXiv:1404.5502] [INSPIRE].

[38] J.B. Dent, F. Ferrer and L.M. Krauss, Constraints on light hidden sector gauge bosons from supernova cooling, arXiv:1201.2683 [INSPIRE]. 
[39] H. Davoudiasl, H.-S. Lee and W.J. Marciano, Dark side of Higgs diphoton decays and muon $g-2$, Phys. Rev. D 86 (2012) 095009 [arXiv: 1208.2973] [INSPIRE].

[40] H. Davoudiasl, H.-S. Lee and W.J. Marciano, 'Dark' Z implications for parity violation, rare meson decays and Higgs physics, Phys. Rev. D 85 (2012) 115019 [arXiv:1203.2947] [INSPIRE].

[41] H. Davoudiasl, H.-S. Lee, I. Lewis and W.J. Marciano, Higgs decays as a window into the dark sector, Phys. Rev. D 88 (2013) 015022 [arXiv:1304.4935] [INSPIRE].

[42] M. Endo, K. Hamaguchi and G. Mishima, Constraints on hidden photon models from electron g- 2 and hydrogen spectroscopy, Phys. Rev. D 86 (2012) 095029 [arXiv:1209.2558] [INSPIRE].

[43] J. Balewski et al., DarkLight: a search for dark forces at the Jefferson Laboratory Free-Electron Laser Facility, arXiv:1307.4432 [INSPIRE].

[44] WASA-AT-COSY collaboration, P. Adlarson et al., Search for a dark photon in the $\pi^{0} \rightarrow e^{+} e^{-} \gamma$ decay, Phys. Lett. B 726 (2013) 187 [arXiv:1304.0671] [INSPIRE].

[45] HADES collaboration, G. Agakishiev et al., Searching a dark photon with HADES, Phys. Lett. B 731 (2014) 265 [arXiv:1311.0216] [InSPIRE].

[46] S. Andreas et al., Proposal for an experiment to search for light dark matter at the SPS, arXiv:1312. 3309 [INSPIRE].

[47] M. Battaglieri et al., The heavy photon search test detector, arXiv:1406.6115 [INSPIRE].

[48] BABAR collaboration, J.P. Lees et al., Search for a dark photon in $e^{+} e^{-}$collisions at BaBar, Phys. Rev. Lett. 113 (2014) 201801 [arXiv:1406.2980] [InSPIRE].

[49] PHENIX collaboration, A. Adare et al., Search for dark photons from neutral meson decays in $p+p$ and $d+A u$ collisions at $\sqrt{s_{N N}}=200 \mathrm{GeV}$, arXiv:1409.0851 [INSPIRE].

[50] D. Kazanas, R.N. Mohapatra, S. Nussinov, V.L. Teplitz and Y. Zhang, Supernova bounds on the dark photon using its electromagnetic decay, Nucl. Phys. B 890 (2015) 17 [arXiv: 1410.0221] [INSPIRE].

[51] A. Blondel et al., Research proposal for an experiment to search for the decay $\mu \rightarrow$ eee, arXiv:1301.6113 [INSPIRE].

[52] R.M. Djilkibaev and R.V. Konoplich, Rare muon decay $\mu^{+} \rightarrow e^{+} e^{-} e^{+} \nu_{e} \bar{\nu}_{\mu}$, Phys. Rev. D 79 (2009) 073004 [arXiv:0812.1355] [InSPIRE].

[53] J. Alwall et al., The automated computation of tree-level and next-to-leading order differential cross sections and their matching to parton shower simulations, JHEP 07 (2014) 079 [arXiv: 1405.0301] [INSPIRE].

[54] Particle Data Group collaboration, K.A. Olive et al., Review of particle physics, Chin. Phys. C 38 (2014) 090001 [inSPIRE].

[55] SuperB collaboration, M. Baszczyk et al., SuperB technical design report, arXiv:1306.5655 [INSPIRE].

[56] BABAR collaboration, B. Aubert et al., The BaBar detector, Nucl. Instrum. Meth. A 479 (2002) 1 [hep-ex/0105044] [inSPIRE].

[57] BABAR collaboration, B. Aubert et al., The BaBar detector: upgrades, operation and performance, Nucl. Instrum. Meth. A 729 (2013) 615 [arXiv:1305.3560] [INSPIRE].

[58] Y. Kuno and Y. Okada, Muon decay and physics beyond the standard model, Rev. Mod. Phys. 73 (2001) 151 [hep-ph/9909265] [INSPIRE]. 